identify and protect all girls and young women who are potential or actual victims of FGM.

\section{G57(P) FOSTERING RESILIENCE: THE PROMOTION OF RESILIENCE IN YOUNG PEOPLE WHO ARE LOOKED AFTER}

A Yeadon, A Share. Department of Community Paediatrics, Leeds Community Healthcare, Leeds, UK

\subsection{6/archdischild-2015-308599.56}

Aims Children and young people who are Looked After have typically experienced significant adversity, with follow up demonstrating poorer social and developmental outcomes than their peers. However, risk factors are not the only predictor of outcomes. Attention is increasingly being focussed on promotion of resilience. There are multiple factors associated with improved resilience. Recurring themes for adolescents include (i) positive relationships with primary carers and with adults outside the family unit; (ii) positive experience of education; (iii) strong social networks, including participation in extra-curricular activities. Here we review the presence of these resilience factors amongst young people in our area.

Methods A questionnaire survey of Looked After young people aged 12-15 years was carried out in 2013. This covered various aspects of home and school life, as well as questions about physical and emotional well-being. Questionnaires were completed by young people either alone or with the support of their school nurse. Out of 84 young people eligible, opportunistic sampling resulted in 38 responses.

Results (i) Adult relationships: 95\% reported that the people looking after them really cared about them, with $89 \%$ able to talk to a parent/carer about their worries. $79 \%$ felt able to talk to an adult who was not their parent/carer. $86 \%$ felt they were taken seriously most of the time.

(ii) Education: 58\% agreed they liked being at school, with $66 \%$ thinking they were doing well at school. $98 \%$ described feeling quite/very safe at school and only $8 \%$ reported bullying within the last year.

(iii) Social networks: All reported having one or more good friends, although only $82 \%$ could talk to friends about their worries. $71 \%$ had taken part in structured extra-curricular activities recently, but $24 \%$ reported there were activities they would like to do but had no opportunity.

Conclusions This survey demonstrates high levels of certain positive resilience factors within our Looked After young people across the three highlighted areas. However, areas for improvement have been identified, including that all young people should have an adult in whom they can confide, and that experience of education is not always a positive one.

\section{G58(P) MULTI-AGENCY REVIEW OF CHILD PROTECTION MEDICAL REPORTS}

IE Wilson, B Lee, D Elliman. Whittington Health NHS Trust, London, UK

10.1136/archdischild-2015-308599.57

The RCPCH Child Protection Companion provides guidance on medical report writing. We wanted to audit local medical reports against this guidance. Reports are shared with social care and police child abuse investigation team (CAIT), contributing to decision making for children. We therefore wished to determine whether our local social care and CAIT teams felt the opinions in the reports were helpful to their decision making.

A retrospective audit of reports of 24 children seen in the first half of 2014 was undertaken, using section 16 of the $\mathrm{RCPCH}$ companion as standards. 3 reports from each of the 8 consultants were chosen at random. CAIT and social care reviewed the same 24 reports and completed a proforma regarding key aspects of the medical opinion.

Patients' details, consultation time/place, specific concerns, appearance of the child, past medical, family and developmental history were completed in all reports. $75 \%$ gave the child's own words where applicable, $89 \%$ gave information regarding school/nursery and $64 \%$ commented on parent/carer interaction. Most reports commented on the need for section 47 investigation but made no direct statement on whether it was safe for the child to return home. Social care and CAIT thought 19 and 16 reports respectively were helpful in decision making. Reported unhelpful factors were non-committal wording on the likelihood of NAI, ambiguity around sibling cases and lack of documentation regarding evidence for neglect concerns.

Clear documentation of patients' and consultation details reflects local strong administrative support. Key areas for child protection, notably the words of the child and child parent interaction were lacking and should be highlighted in training. Paediatricians may have made verbal recommendations during the medical but documentation of this is imperative. The satisfaction of CAIT/social care with reports depends upon their expectations as well as the doctor's clarity. In some cases the doctor could not have given a 'clear' opinion based on what was in front of them. It is valuable for local audits of child protection reports to involve local CAIT and social care to optimise the usefulness of reports in safeguarding of children.

\section{British Society of Paediatric Dermatology and British Paediatric Neurology Association}

\section{G59 THE INTERNATIONAL COLLABORATIVE INFANTILE SPASMS STUDY (ICISS) COMPARING HORMONAL THERAPIES (PREDNISOLONE OR TETRACOSACTIDE DEPOT) AND VIGABATRIN VERSUS HORMONAL THERAPIES ALONE IN THE TREATMENT OF INFANTILE SPASMS: EARLY CLINICAL OUTCOME}

${ }^{1} \mathrm{~F}$ O'Callaghan, ${ }^{2,3} \mathrm{~S}$ Edwards, ${ }^{3} \mathrm{E}$ Hancock, ${ }^{4} \mathrm{~A}$ Johnson, ${ }^{5} \mathrm{C}$ Kennedy, ${ }^{6} \mathrm{~A}$ Lux, ${ }^{7} \mathrm{M}$ Mackay, ${ }^{8} \mathrm{R}$ Newton, ${ }^{9} \mathrm{M}$ Nolan, ${ }^{10} \mathrm{D}$ Rating, ${ }^{11} \mathrm{~B}$ Schmitt, ${ }^{12} \mathrm{C}$ Verity, ${ }^{2,3} \mathrm{~J}$ Osborne. ${ }^{1} \mathrm{Clinical}$ Neurosciences, UCL, London, UK; ${ }^{2}$ Department for Health, University of Bath, Bath, UK; ${ }^{3}$ Children's Centre, Royal United Hospital, Bath, UK; ${ }^{4}$ MRC Biostatistics Unit, University of Cambridge, Cambridge, UK; ${ }^{5}$ Faculty of Medicine, University of Southampton, Southampton, UK; ${ }^{6}$ Paediatric Neurology, Bristol Royal Hospital for Children, Bristol, UK; ${ }^{7}$ Paediatric Neurology, Royal Children's Hospital, Melbourne, Australia; ${ }^{8}$ Paediatric Neurology, Royal Manchester Children's Hospital, Manchester, UK; ${ }^{9}$ Paediatric Neurology, Starship Children's Hospital, Auckland, New Zealand; ${ }^{10}$ Neuropaediatrics, University of Heidelberg, Heidelberg, Germany; ${ }^{11}$ Neuropaediatrics, University Children's Hospital, Zurich, Switzerland; ${ }^{12}$ Paediatrics, Addenbrooke's Hospital, Cambridge, UK

\subsection{6/archdischild-2015-308599.58}

Infantile spasms (IS) are a serious epileptic encephalopathy that occur most commonly between 2 and 14 months. The spasms occur in association with hypsarrhythmia or similar on EEG. There is often coincident psychomotor arrest or regression. Between March 2007 and May 2014, infants with IS and a compatible EEG were enrolled in a multicenter treatment trial. 